\title{
AN EFFICIENT PATIENT INFLOW PREDICTION MODEL FOR HOSPITAL RESOURCE MANAGEMENT
}

\author{
Kottalanka Srikanth ${ }^{1}$ and D. Arivazhagan ${ }^{2}$ \\ Department of Information Technology, AMET University, India
}

\begin{abstract}
There has been increasing demand in improving service provisioning in hospital resources management. Hospital industries work with strict budget constraint at the same time assures quality care. To achieve quality care with budget constraint an efficient prediction model is required. Recently there has been various time series based prediction model has been proposed to manage hospital resources such ambulance monitoring, emergency care and so on. These models are not efficient as they do not consider the nature of scenario such climate condition etc. To address this artificial intelligence is adopted. The issues with existing prediction are that the training suffers from local optima error. This induces overhead and affects the accuracy in prediction. To overcome the local minima error, this work presents a patient inflow prediction model by adopting resilient backpropagation neural network. Experiment are conducted to evaluate the performance of proposed model inter of RMSE and MAPE. The outcome shows the proposed model reduces RMSE and MAPE over existing back propagation based artificial neural network. The overall outcomes show the proposed prediction model improves the accuracy of prediction which aid in improving the quality of health care management.
\end{abstract}

Keywords:

Artificial Inteligence, Forecasting, Optimization, Prediction

\section{INTRODUCTION}

There has been increase in demand to manage hospital resource for providing efficient services to the end user/patient. Providing better healthcare service to its patient and minimizing hospital resources [1] is a key feature. Many hospitals across globe operate under severe resources constraint. The hospital are bound to provides admission to emergency patient and provide the best medication care as possible. The inability to treat emergency patient due to resource constraint (example: shortage of bed) has led wide criticism from public to government agencies [2]. The hospital also operates under strict budgetary constraint. Therefore need an effective management technique to manage these resources.

The biggest concern of hospital management is the admittance of emergency patient in presence of pre-appointed patient. Due to improper prediction of emergency patient inflow will lead to congestion or blockade in service [3]. The hospital has to determine the number of pre-appointed patient to be given admission in order to provide admittance to the emergency patient. The hospital consist of various department such diabetes test, blood test, CT-scan, X-ray, surgeries etc. which are generally crowded due to improper queuing management.

Decision making strategy for optimal admittance of preappointed patient is need to be done in advance which ranges from weeks to month. To schedule patient and arrange surgeries requires the technician to be trained in advance. Therefore due to these constraints there is a necessity to develop a forecasting model to predict the patient inflow.

Recently there has been many pre-hospital emergency medical services (EMS) model to handle emergency cases has been presented [4]-[6]. Among most the Out of Hospital Cardiac Arrest (OHCA) has the least survival rate which requires timely medical care. The Table. 1 shows how the response time is so critical in improving survival rate.

Table.1. Response time and Survival rate

\begin{tabular}{|c|c|c|c|}
\hline Response time & $\begin{array}{c}\text { 72 Hours } \\
\text { Survival }\end{array}$ & $\begin{array}{c}\text { Number } \\
\text { of Event }\end{array}$ & $\begin{array}{c}\text { Survival } \\
\text { rate (\%) }\end{array}$ \\
\hline 0-3 minutes & 30 & 172 & 17.44 \\
\hline 3-6 minutes & 129 & 1243 & 10.38 \\
\hline 6-10 minutes & 91 & 91 & 11.55 \\
\hline >10 minutes & 12 & 12 & 6.03 \\
\hline
\end{tabular}

The transportation and rescue strategy play an important part in handling critical patient such as stroke [7] and trauma [8]. Ambulance dispatch service [9] play a critical role in improving patient care. That is the faster the ambulance reach the incident the better the chances of survival. Many optimization strategies have been presented in [10], [11] and [12]. Therefore reducing the response time and short-term forecasting plays a significant role in managing hospital resources more efficiently.

Many patient inflow predictions based on queue [13], Game theory [14], Markov [15], [16] and [17] and time series based model has been presented in [18] that forecast in hour and daily basis. In [19] assessed the accuracy of volume prediction by using demand pattern and compared smoothing average and average peak demand and also highlighted the factor that will aid in improving accuracy of prediction model. In [20] presented a prediction model by adopting moving average namely ARIMA model. The ARIMA predicts the demand on daily basis considering the weather factor. In [21] and [22] adopted back propagation neural network for prediction of EMS demand and compared neural network with moving average. The outcome shows that the neural network outperforms Moving average in every aspect. They considered only limited feature (4 features) and they suffer from local optima error [23].

The main issue in training backpropagation neural network is in what rate the weights are updated. The other problem of Back propagation algorithm is that when there is an optimization 
problem present in updating local minima. This will induce an error in prediction i.e. when there is high likelihood of decrease in updating/minimizing local minima the root selected end up in a situation where they cannot reduce anymore (getting stuck at local optima), and it never search for new root i.e. it will never able to jump out of local optima.

To overcome this issue, an efficient method of searching the global minima by using the Resilient Backpropagation technique that aids in improving the accuracy of our patient inflow prediction model is adopted.

The paper organization is as follows: The literature survey is described in section 2. In section 3 the proposed Patient Inflow Prediction model is presented. The penultimate section presents a simulation and experimental study. The concluding and future work is discussed in the last section.

\section{LITERATURE SURVEY}

There is an increase demand of prediction model for various application uses in managing hospital resources. Since the prediction play a significant role in improving patient care service. Recently there has been wide research been developed to manage hospital resources by adopting time series, linear model and artificial intelligence and so on which is surveyed below.

The significance of neural network methodology used for kidney transformation in patients is discussed in model [24]. Here they discussed about the complications occurs in commonly used ARMA Model and to overcome these nonlinear complications Artificial Neural Network come under existence. In this current era, need of precise concentric dose of Cyclosporine in the kidney transformation has forced to use more refined and accurate methodologies like Artificial Neural Network which provides exceptional outcomes. This model discusses about its types, its methodology, issues related to traditional ARMA model and results obtained from the prediction. ANN consists of three type of networks, one is Multilayer Perceptron (MLP) modeling [25], second is finite impulse response modelling [26] and third is Elman recurrent [27]. The complete model is carried out in Matlab. With graphical representation this model concludes about the precision and robustness of Artificial Neural methodologies compare to traditional ARMA models.

The significance of this model [28] is based on the analysis of previously collected data to provide better emergency facilities to needy people. To obtain the required outcomes geographical information system methodology has been proposed in their work. Multiple methodologies like moving average, artificial neural network [30] and support vector methods are used for the analysis of collected data in specified locality with integration of GIS technology. They emphasize on backward propagation [31] with the integration of ANN to provide proper data analysis with time and space. They concluded the forecast as $23.01 \%$ which can be considered as reasonable [32] so that based on these results for specific required locality better pre emergency facilities can be provided.

In [33] emphasizes on issues encountered by hospitals related to controlling of queue due to overpopulation. Avoidable interruptions for an excessive time make people irritated and exasperated. To avoid this type of issues they provided a real-time application by which patients can get information about their well- organized cure idea and forecast waiting time using the patient treatment time prediction algorithm. For this purpose they have used patient's real time data from different hospitals to model the cure time of patient's .Based on this collected data the cure time of every recipient is forecasted and for which hospital queuing recommendation (HQR) service is established. All the collected data is employed on cloud using APACHE platform. Using Classification \& Regression tree prototype with the integration of random forest algorithm all the Big Data is used for well-organized cure of patients is proposed in this prototype.

In this modern era, demand is much larger than the supply. In [34] emphasizes on issues related to prediction of load (power) in large localities like big hospitals and how these issues can be addressed to provide better supply according to the vital demands. To achieve this purpose of load prediction they proposed the Artificial Neural Network technology with the integration of backpropagation algorithm. Load prediction can give the facts \& figures for the advancement of energy for small and moderate energy sharing systems. ANN design modeled in this paper uses multilayer perceptron arrangement for load prediction. The complete prototype is carried out in Matlab environment. The outcomes of this analysis are adequate with realistic errors. Based on these experimental results, this model can be implemented for the built-up and local purposes.

The essential to minimize the rising cost of care through ensuring convenience of rare health resources and optimum utilization is effective resource necessity forecasting [35]. Covariates are used for the better in hospital capability planning and it will help the patient's predictable destination after release. Many of deterministic model have failed to inherent changeability in the complex health methods e.g. ratio-based methods. This paper considering more number of absorbing state with Markovprocess, then it describe how the model is helpful for valuing cost of care, social, community care source necessity and admission planning [36]. A clinical decision support (CDS) system is presented for the study of heart failure patients. It gives the output such as, heart failure (HF) type prediction, HF severity evaluation, and a management interface which help to compares different patients continuation. A machine learning method is adopted for the implementation of smart intelligent functions. The randomforest method (RFM) is used for the evaluation of best performance between hear failure (HF) type prediction function and hear failure (HF) severity. Several machines learning method (MLM) are comparing to give these outputs, but it is problematic to simplify these finding because of small sample size.

A number of disease risk valuation with uniform prototype is shown in this paper [37]. Formless clinical medical text is the important part of electronics health records, it is characterized through the large quantities and it can store considerable disease related info of patients. It is difficult to use risk valuation prototype in all disease application. Learning methods study and excerpt the disease characteristics can be used to measure disease risks. The different diseases risk valuation with same erection, the approach is projected in this article.

The main financial concern is blood product transfusion for the patients and hospital. In [22] and [38] evaluates the effectiveness of using (ANNs) artificial neural networks aimed at forecast the transfusion requirements for the trauma patients. Effective utilization of resources is a crucial problem if minimizing costs 
with maximizes patient care at hospital. In this domain the traditional statistical prototype do not doing well. An additional problem is speed with which difficult decisions and preparation can be made. Artificial neural networks (ANNs) can predict accurately most of the emergency room (ER) patient transfusion requirements. The further research is needed to examine heterogeneous ANNs model for the multiple populations.

The overall survey shows that artificial intelligence plays a significant role in improving prediction accuracy than other linear regression model. However, it suffers from minimizing the local minima error. To overcome this, here an efficient global searching mechanism is considered by adopting resilient back propagation for forecasting future inflow of patient which is presented in next section.

\section{PROPOSED PREDICTION}

Data Preprocessing: In this stage the hospital patient inflow data for different department considering inpatient and outpatient are gathered. Every day numerous numbers of patients visit the hospital for various treatment purposes. Let $Z$ be the set of patient in a hospital and the patient that has registered its information is depicted by $z_{n}$. Let consider that there are $H$ patient in $Z$ which is as follows:

$$
Z=\left\{z_{1}, z_{2}, \ldots, z_{H}\right\}
$$

where, each individual/patient $z_{n}$ has parameters/attributes such as id, age, gender and so on. Each patient can visit to multiple departments for various checkups. Let $C \mid z_{n}$ be the collection of clinical diagnosis performed on patient $z_{n}$ in his stay as follows:

$$
C \mid z_{n}=\left\{c_{1}, c_{2}, \ldots, c_{L}\right\}
$$

where, each clinical diagnosis data $c_{1}$ consist of information $I$ such as department, number of days, doctor and staff attended and so on is as follows:

$$
I \mid z_{n}=\left\{i_{1}, i_{2}, \ldots, i_{G}\right\}
$$

where, $i_{j}$ is the feature param of patient clinical diagnosis $z_{n}$. Here the patient inflow for different department value for each day is obtained and patient inflow is predicted using supervised machine learning using these data.

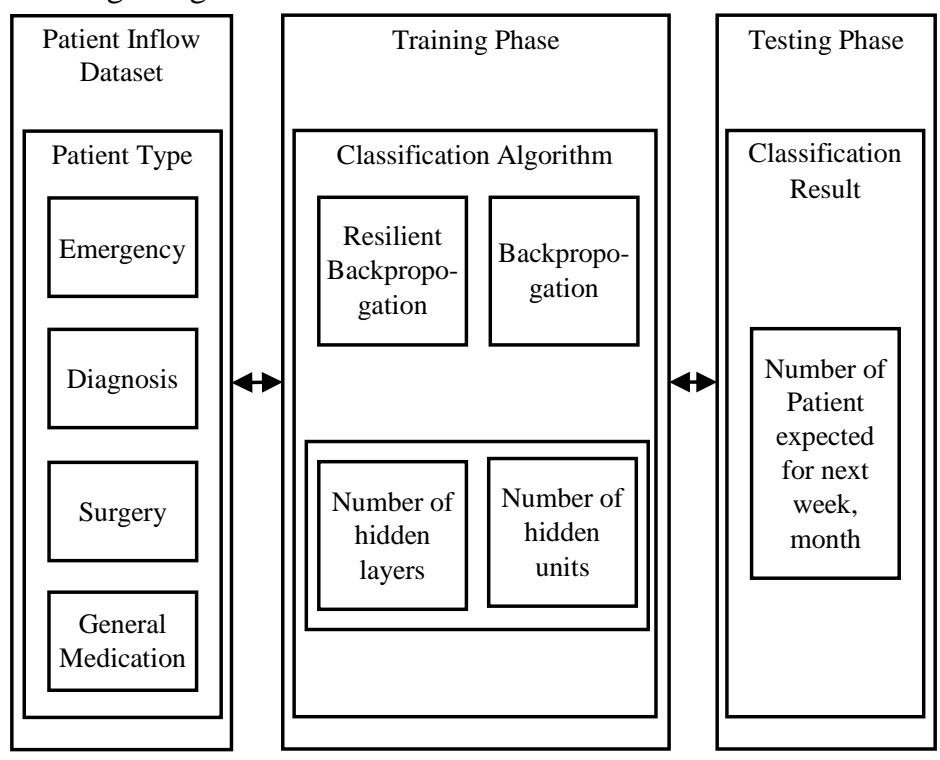

Fig.1. Architecture of patient inflow prediction model
The architecture of proposed patient inflow prediction model is shown below Fig.1. Here backpropagation for training data of Patient Inflow Prediction (PIP) is adopted. The basic step of a simple backpropagation learning model is to update the network weights and biases in direction of rapidly decreasing objective function, with negative gradient. The single iteration of backpropagation is computed as follows:

$$
a_{s+1}=a_{s}-\gamma_{s} d_{s}
$$

where, $\gamma_{s}$ is the learning rate, $a_{s}$ is the present biases and weights vector and $d_{s}$ is the present gradient. The gradient can be computed by following methods.

In batch method, the weight is updated once all the input data is applied to the network were as in incremental approach the weights are updated and gradient is computed after every input data is applied to the network. In batch steepest descent approach the biases and weights are simplified in direction of objective functions negative descent. Similarly in batch method network biases and weights are updated after applying entire training data to a network. Therefore, as long as the methods have net input, weights and transferal param have derivative function the batch gradient descent approach without momentum can be used to train any network. The derivative of objective param $O$ for weight and biases $A$ can be computed using backpropagation neural network as follows:

$$
\partial A=p_{q} \frac{\partial O}{\partial A}
$$

where, $p_{q}$ is the learning rate. The training ends when following circumstances is met, when the determined period of time is met, the objective function has minimized the training objectives, the gradient objective pram falls below minimum objective gradient param, when maximum number of iteration is reached and lastly when verification objective increases the maximum verification failures param. Gradient descent with momentum is a backpropagation network training approach that updates biases and weights as per gradient decent with momentum. Therefore as long as it has net input, weights and transferal param the model can be trained using backpropagation. The derivative of objective param $O$ for weight and biases param $A$ can be computed using backpropagation neural network with momentum as follows:

$$
\partial A=t_{x} \partial A_{O}+p_{q}\left(1-t_{x}\right) \frac{\partial O}{\partial A}
$$

where, $t_{x}$ is the constant of momentum, $p_{q}$ is the learning rate of network, $\partial O$ is the preceding modified biases or weight. The training ends when following circumstances is met, when the determined period of time is met, the objective function has minimized the training objectives, the gradient objective param falls below minimum objective gradient param, when maximum number of iteration is reached and lastly when verification objective increases the maximum verification failures param.

The hidden layer in multilayer network uses sigmoid transferal methods. These methods are generally called as the squashing methods. As the results are finite outputs by compressing a given input. The characteristic computation of sigmoid methods is that as inputs get higher the slopes must come down to zero which is problematic. Especially when using steepest descent in training a multilayer sigmoid methods. Since the gradient can have tiny magnitude, as a result have impact on biases and weight. 
To address this, resilient back propagation neural network for training PIP data is used. The resilient algorithms are used to remove the impact of magnitude of the derivative functions.

Here the derivative magnitude has no impact on updating weight as only the sign of derivative used to show the direction of updating weight. A separate update param is used to determine size of weight. Whenever the objective function derivatives with respect to weight that has same param in two iterations, the update param of every bias and weight is improved by some element. As long as it has net input, weights and transferal param the resilient backpropagation can train a network. The derivative of objective param $O$ for weight and biases param $A$ can be computed using backpropagation neural network as follows:

$$
\partial A=\delta A \times \operatorname{param}\left(d_{A}\right)
$$

where, $d_{A}$ is the gradient and the component of $\delta A$ are initially set to zero. In each and every epoch the component of $\delta A$ is updated/changed. If a component of $d_{A}$ is same after consecutive epoch, then the component of $\delta A$ is increased by $\delta_{i n c}$. If a component of $d_{A}$ param changes from consecutive epoch then the resultant component of $\delta A$ is decreased by $\delta_{d e c}$. To evaluate the prediction error and accuracy of our approach, this paper uses Mean Absolute Percentage Error (MAPE) and Root mean square error (RMSE). The MAPE and RMSE is computed as follows:

$$
\begin{gathered}
M A P E=\frac{1}{E} \sum_{v=1}^{E}\left|\frac{f_{v}-a_{v}}{a_{v}}\right| \times 100 \\
R M S E=\sqrt{\frac{1}{E} \sum_{v=1}^{E} a_{v}-f_{v}}
\end{gathered}
$$

where, $a_{v}$ is the actual parameter, $f_{v}$ is the predicted parameter and $E$ is the total number of tuples/days to estimate.

\section{SIMULATION RESULT AND ANALYSIS}

The simulation is conducted to evaluate the performance of proposed patient inflow prediction model. The system environment used windows 10 pro 64 bit operating system, Intel Pentium i5 class processor, 8GB of RAM. The model is designed using visual studio dot net framework 4.0, 2010 and c\# programming language. The experiment is conducted using datasets [39] and [29]. The predication model accuracy is evaluated interm of root mean square error and Mean Absolute Percentage Error considering different data sets. A comparison of existing back propagation and proposed resilient back propagation is given.

The dataset [39] consist of patient inflow admission from April 2008 to June 2010. Firstly both the model is trained for each month of 2009 and prediction is done for years 2010. The Fig. 2 shows the prediction of patient inflow for each month of year 2010. The outcomes show the proposed prediction model outperforms the existing model. The overall RMSE is performance is shown in Fig.3. The outcome shows that the RMSE of proposed prediction model is reduced by $29.63 \%$ and MAPE is reduced by $79.29 \%$ over existing model which is shown in Fig.3 and Fig.4 respectively.

To consider a complex scenario for prediction performance of our approach we consider the following dataset [29] which consist of patient inflow data of various departments in a hospital from year June 2011 to May 2015. The input data consist of number of patient register per month for each department. The first year of patient inflow data is trained for both existing and proposed approach considering and the next four years patient inflow data is predicted. The Fig.5, Fig.6, Fig.7 and Fig.8 shows the Patient inflow prediction of emergency, Diagnosis, General and Surgery patient of both proposed and existing approach respectively. The outcome shows that the proposed approach outperform existing approach. The RMSE of proposed model is reduced by $31.77 \%$ and MAPE is reduced by $88.24 \%$ over existing model which is shown in Fig. 9 and Fig.10 respectively.

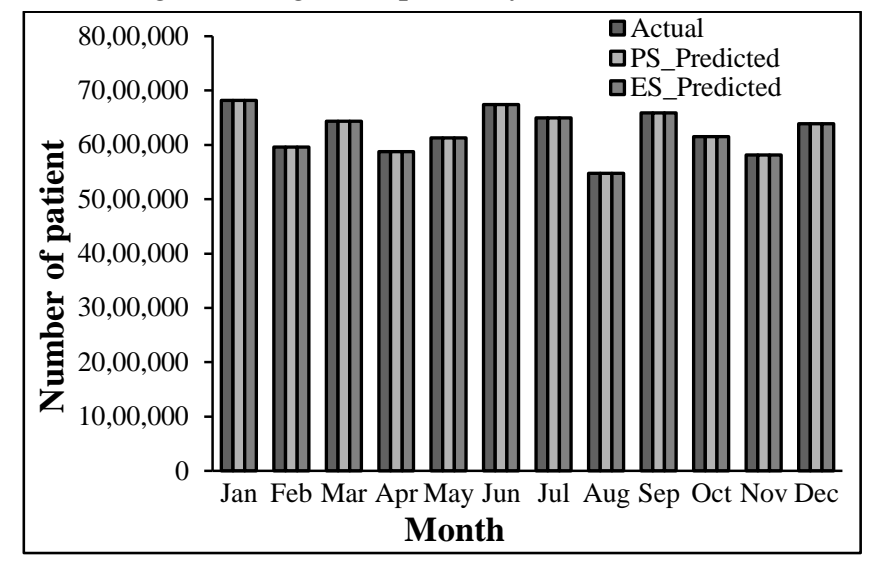

Fig.2. Patient inflow prediction for year 2010

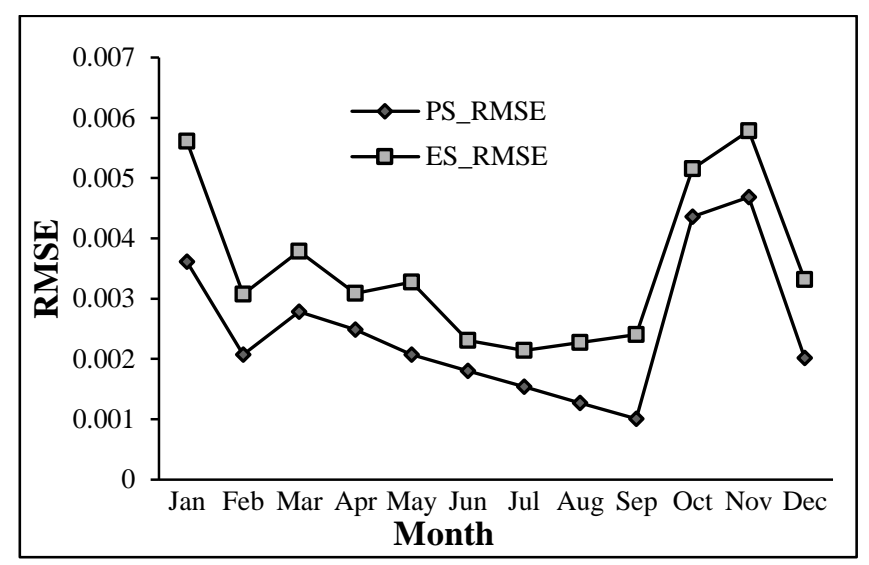

Fig.3. RMSE performance of patient inflow prediction for year 2010

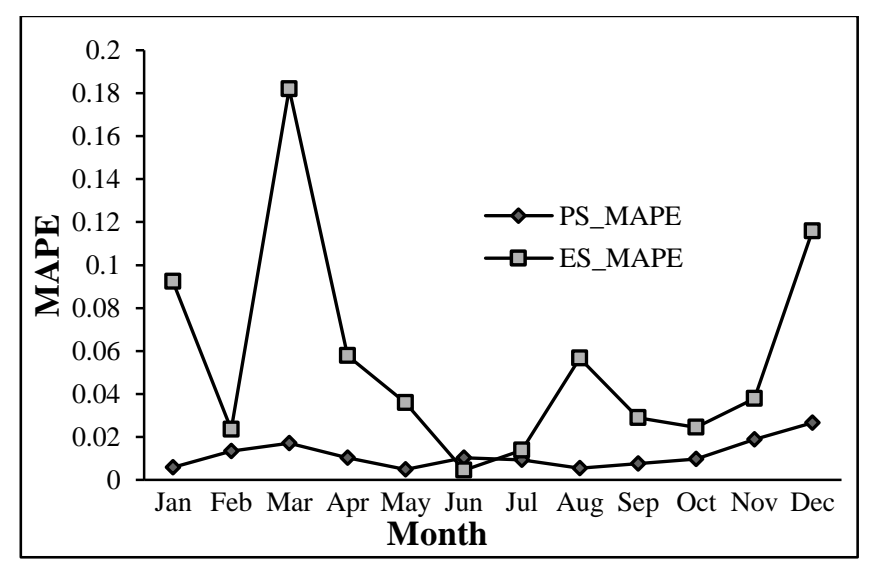

Fig.4. MAPE performance of patient inflow prediction for year 2010 


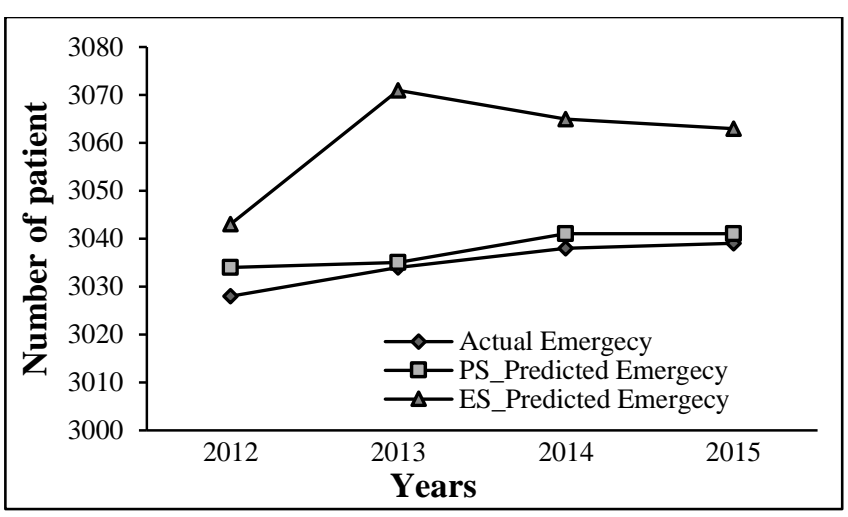

Fig.5. Patient inflow Prediction of Emergency Patient

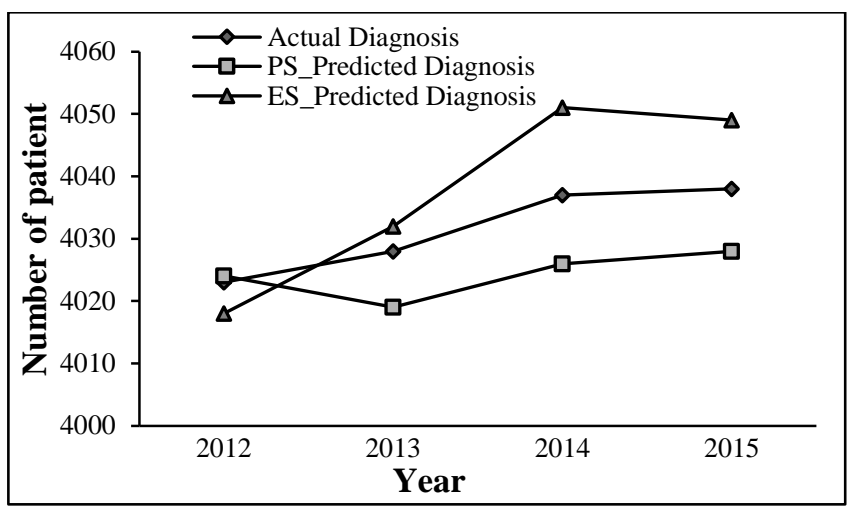

Fig.6. Patient inflow Prediction of Diagnosis Patient

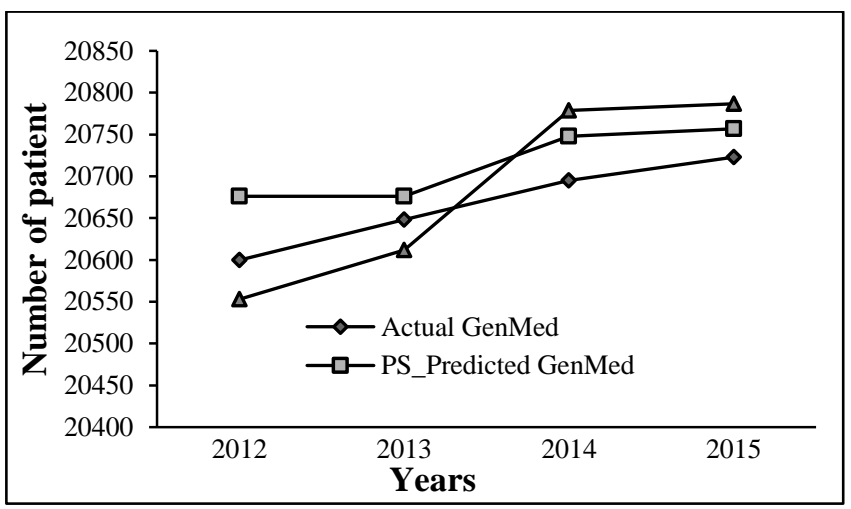

Fig.7. Patient inflow Prediction of General Patient

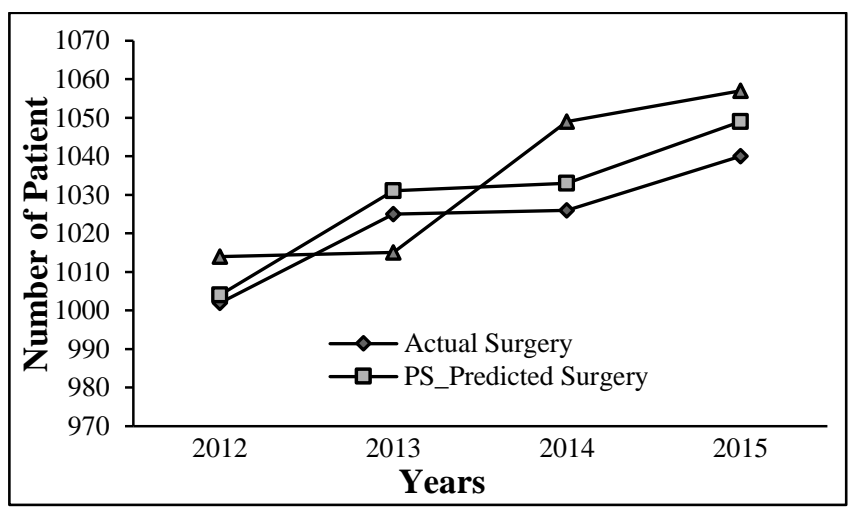

Fig.8. Patient inflow Prediction of Surgery Patient

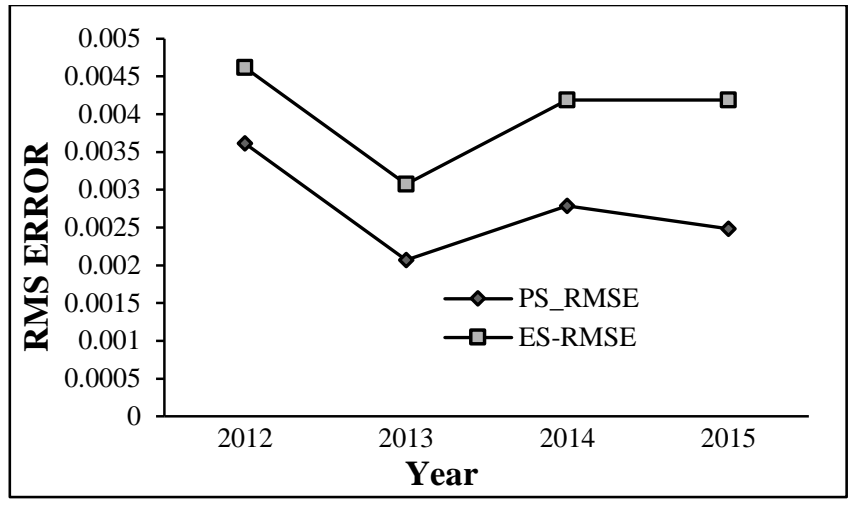

Fig.9. RMSE performance of Patient inflow Prediction

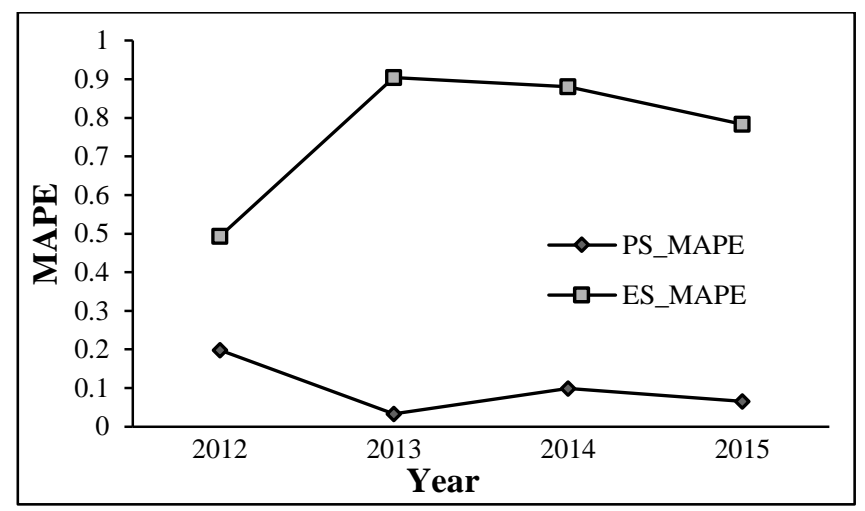

Fig.10. MAPE performance of Patient inflow Prediction

\section{CONCLUSION}

This work presents an optimization strategy for predicting patient inflow in hospital. The model considered predicting patient inflow for different department. This aids in utilizing hospital resources in efficient manner. A multi-layer artificial neural network perception by adopting resilient back propagation is considered. Simulation is conducted to evaluate the performance of patient inflow prediction model. The accuracy of prediction model is evaluated considering performance of RMSE and MAPE. Simulation is conducted for both proposed and existing model for varied datasets shows significant performance improvement of proposed prediction model over exiting prediction model. The overall result shows the proposed patient inflow prediction model is efficient when compared to model in [33], [34] and [37] that adopted artificial intelligence. The result outcome obtained pushes the proposed model to forecast other types of dataset such as ambulance prediction for scheduling, disease, medical risk assessment prediction for diagnosis and so on. In future more features for training is considered to improve the prediction model.

\section{REFERENCES}

[1] S. Ivatts and P. Millard, "Health Care Modelling: Why Should We Try?", British Journal of Health Care Management, Vol. 8, No. 6, pp. 218-222, 2002.

[2] Victorian Public Hospitals, "Performance Monitoring Framework, Available at: https://www2.health.vic.gov.au/hospitals-and-health- 
services/funding-performance-accountability/performancemonitoring.

[3] D.M. Fatovich, Y. Nagree and P. Sprivulis, “Access Block causes Emergency Department Overcrowding and Ambulance Diversion in Perth, Western Australia", Emergency Medicine Journal, Vol. 22, No. 5, pp. 351-354, 2005.

[4] K. Peleg and J.S. Pliskin, "A Geographic Information System Simulation Model of EMS: Reducing Ambulance Response Time", The American Journal of Emergency Medicine, Vol. 22, No. 3, pp. 164-170, 2004.

[5] F.L. Henriksen, P. Schorling, B. Hansen, H. Schakow and M.L. Larsen, "FirstAED Emergency Dispatch, Global Positioning of First Responders with Distinct Roles - A Solution to Reduce the Response Times and Ensuring Early Defibrillation in the Rural Area Langeland", Proceedings of International Conference on Well-Being in the Information Society, pp. 36-45, 2014.

[6] J. Fitch, "Response Times: Myths, Measurement and Management", Journal of Emergency Medical Services, Vol. 30, No. 9, pp. 47-56, 2005.

[7] S.A. Simonsen, M. Andresen, L. Michelsen, S. Viereck, F.K. Lippert and H.K. Iversen, "Evaluation of Pre-Hospital Transport Time of Stroke Patients to Thrombolytic Treatment", Scandinavian Journal of Trauma, Resuscitation and Emergency Medicine, Vol. 22, No. 65, pp. 1-5, 2014.

[8] A. Timma, M. Maegelea, R. Leferingb, K. Wendtc and H. Wyend, "Pre-Hospital Rescue Times and Actions in Severe Trauma. A Comparison between Two Trauma Systems: Germany and the Netherlands", Injury, Vol. 45, No. 3, pp. 43-52, 2014.

[9] Shane G. Henderson and Andrew J. Mason, "Ambulance Service Planning: Simulation and Data Visualisation", International Series in Operations Research and Management Science, Vol. 70, pp. 77-102, 2004.

[10] Cheng Siong Lim, Rosbi Mamat and Thomas Braunl, "Impact of Ambulance Dispatch Policies on Performance of Emergency Medical Services", IEEE Transactions on Intelligent Transportation Systems, Vol. 12, No. 2, pp. 624632, 2011.

[11] N. Geroliminis, M.G. Karlaftis and A. Skabardonis, "A spatial queuing model for the emergency vehicle districting and location problem", Transportation Research Part B: Methodological, Vol. 43, No. 7, pp. 798-811, 2009.

[12] H.K. Rajagopalan, "Ambulance Deployment and Shift Scheduling: An Integrated Approach”, Journal of Service Science and Management, Vol. 4, No. 1, pp. 66-78, 2011.

[13] Elif Akcali, Murray J. Cote and Chin Lin, "A Network Flow Approach to Optimizing Hospital Bed Capacity Decisions", Health Care Management Science, Vol. 9, No. 4, pp. 391404, 2006.

[14] R. Zongwei, L. Chuanqing and G. Haini, "Strategy on Doctor Resource Sharing among Hospitals composed Regional Medical Association based on Game Theory", Proceedings of $3^{\text {rd }}$ International Conference on Information Management, pp. 274-278, 2017.

[15] H. Xie T.J. Chaussalet and P.H. Millard, "A Model-based Approach to the Analysis of Patterns of Length of Stay in Institutional Long-Term Care", IEEE Transactions on
Information Technology in Biomedicine, Vol. 10, No. 3, pp. 512-518, 2006.

[16] Xiao Chen, Liangmin Wang, Jie Ding and Nigel Thomas, "Patient Flow Scheduling and Capacity Planning in a Smart Hospital Environment", IEEE Access, Vol. 4, pp. 135-148, 2016.

[17] H. Xu, W. Wu, S. Nemati and H. Zha, "Patient Flow Prediction via Discriminative Learning of MutuallyCorrecting Processes", Proceedings of $33^{\text {rd }}$ International Conference on Data Engineering, pp. 37-38, 2017.

[18] N. Channouf, P. L'Ecuyer, A. Ingolfsson and A.N. Avramidis, "The Application of Forecasting Techniques to Modeling Emergency Medical System Calls in Calgary, Alberta”, Health Care Management Science, Vol. 10, No. 1, pp. 25-45, 2007.

[19] Lawrence H. Brown, E. Brooke Lerner, Baxter Larmon, Todd Le Gassick and Michael Taigman, "Are EMS Call Volume Predictions based on Demand Pattern Analysis Accurate?", Prehospital Emergency Care, Vol. 11, No. 2, pp. 199-203, 2007.

[20] Ho-Ting Wong and Poh-Chin Lai, "Weather Factors in the Short-Term Forecasting of Daily Ambulance Calls", International Journal of Biometeorology, Vol. 58, No. 5, pp. 669-678, 2014.

[21] H. Setzler, C. Saydam and S. Park, "EMS Call Volume Predictions: A Comparative Study", Computers and Operations Research, Vol. 36, No. 6, pp. 1843-1851, 2009.

[22] Riad Alharbey, "Predictive Analytics Dashboard for Monitoring Patients in Advanced Stages of COPD", Proceedings of $49^{\text {th }}$ Hawaii International Conference on System Sciences, pp. 3455-3461, 2016.

[23] Debasish Basak, Srimanta Pal and Dipak Chandra Patranabis, "Support Vector Regression", Neural Information Processing-Letters and Reviews, Vol. 11, No. 10, pp. 203-224, 2007.

[24] G. Camps Valls et al., "Prediction of Cyclosporine Dosage in Patients after kidney Transplantation using Neural Networks", IEEE Transactions on Biomedical Engineering, Vol. 50, No. 4, pp. 442-448, 2003.

[25] A.S. Weigend and N.A. Gershenfeld, "Time Series Prediction. Forecasting the Future and Understanding the Past", Proceedings of Advanced Research Workshop on Comparative Time Series Analysis, pp. 279-313, 1992.

[26] E.A. Wan, "Finite Impulse Response Neural Networks with Applications in Time Series Prediction”, Ph.D. Dissertation, Department of Electrical Engineering, Stanford University, 1993.

[27] Jeffrey L. Elman, "Finding Structure in Time", Cognitive Science, Vol. 14, No. 2, pp.179-211, 1988.

[28] A.Y. Chen, T.Y. Lu, M.H.M. Ma and W.Z. Sun, "Demand Forecast using Data Analytics for the Preallocation of Ambulances", IEEE Journal of Biomedical and Health Informatics, Vol. 20, No. 4, pp. 1178-1187, 2016.

[29] HES Outpatient dataset, Available at: http://www.adls.ac.uk/hscic/nhs-hes/?detail.

[30] H. Setzler, C. Saydam and S. Park, "EMS Call Volume Predictions: A Comparative Study", Computers and Operations Research, Vol. 36, No. 6, pp. 1843-1851, 2009.

[31] C.M. Bishop, "Pattern Recognition and Machine Learning", Springer, 2006. 
[32] C.D. Lewis, "Industrial and Business Forecasting Methods: A Practical Guide to Exponential Smoothing and Curve Fitting", Butterworth Scientific, 1982.

[33] J. Chen, K. Li, Z. Tang, K. Bilal and K. Li, "A Parallel Patient Treatment Time Prediction Algorithm and Its Applications in Hospital Queuing-Recommendation in a Big Data Environment", IEEE Access, Vol. 4, pp. 1767-1783, 2016

[34] A. Bagnasco, M. Saviozzi, F. Silvestro, A. Vinci, S. Grillo and E. Zennaro, "Artificial Neural Network Application to Load Forecasting in a Large Hospital Facility", Proceedings of International Conference on Probabilistic Methods Applied to Power Systems, pp. 1-6, 2014.

[35] L. Garg, S.I. McClean, M. Barton, B.J. Meenan and K. Fullerton, "Intelligent Patient Management and Resource Planning for Complex, Heterogeneous, and Stochastic Healthcare Systems", IEEE Transactions on Systems, Man, and Cybernetics-Part A: Systems and Humans, Vol. 42, No. 6, pp. 1332-1345, 2012.

[36] G. Guidi, M.C. Pettenati, P. Melillo and E. Iadanza, “A Machine Learning System to Improve Heart Failure Patient Assistance", IEEE Journal of Biomedical and Health Informatics, Vol. 18, No. 6, pp. 1750-1756, 2014.

[37] X. Shi, Y. Hu, Y. Zhang, W. Li, Y. Hao, A. Alelaiwi, S.M.M. Rahman and M.S. Hossain, "Multiple Disease Risk Assessment with Uniform Model based on Medical Clinical Notes", IEEE Access, Vol. 4, pp. 7047-7083, 2016.

[38] S. Walczak, “Artificial Neural Network Medical Decision Support Tool: Predicting Transfusion Requirements of ER Patients", IEEE Transactions on Information Technology in Biomedicine, Vol. 9, No. 3, pp. 468-474, 2005.

[39] Hospital Episode Statistics (HES): Outpatient ActivityProvider-level analysis, Available at: http://data.gov.uk/dataset/hospital_outpatient_activity. 